\title{
A.Chancerel, C.Marcigny, et E.Ghesquière (dir.), Le plateau de Mondeville (Calvados) : du Néolithique à l'âge du Bronze
}

Jean-Laurent Monnier

\section{CpenEdition}

\section{Journals}

Édition électronique

URL : http://journals.openedition.org/rao/484

DOI : $10.4000 /$ rao.484

ISBN : 978-2-7535-1607-6

ISSN : 1775-3732

Éditeur

Presses universitaires de Rennes

\section{Édition imprimée}

Date de publication : 30 décembre 2007

ISBN : 978-2-7535-0574-2

ISSN : 0767-709X

\section{Référence électronique}

Jean-Laurent Monnier, «A.Chancerel, C.Marcigny, et E.Ghesquière (dir.), Le plateau de Mondeville (Calvados) : du Néolithique à l'âge du Bronze », Revue archéologique de l'Ouest [En ligne], 24 | 2007, mis en ligne le 30 décembre 2009, consulté le 06 décembre 2020. URL : http://journals.openedition.org/ rao/484; DOI : https://doi.org/10.4000/rao.484 
La publication s'ouvre par quelques contributions introductives sur les contextes culturels (émergence de la métallurgie), chronologiques (avec une fourchette de quelque deux millénaire entre le début des productions bulgares et la fin de celles du Grand-Pressigny), sociologiques (« les objets qui voyagent, les sociétés qui échangent ») et technologique (présentation en parallèle des deux techniques de débitage en cause : la pression et la percussion indirecte). Après quoi, l'on passe à la présentation des quatre ensembles retenus, de leur production et de leur diffusion.

Suit un catalogue des objets présentés : 103 pour la Bulgarie, 29 pour l'Ukraine, 72 pour l'Andalousie et 244 pour la Touraine, puis une précieuse bibliographie judicieusement organisée en bibliographie " générale " au sens large (elle-même subdivisée) et en bibliographies régionales (une centaine de références au total). L'illustration, très largement en couleurs, est de belle qualité, la mise en page moderne et agréable et, c'est à noter, le texte des articles est intégralement traduit en anglais.

Une réflexion vient à l'examen des photographies présentées : la fameuse couleur "blond-cire " du silex pressignien, même si elle n'est pas aussi omniprésente qu'on l'a parfois prétendu, est néanmoins dominante; or cette teinte chaude se retrouve en Bulgarie et, pour partie, en Andalousie (mais apparemment pas en Ukraine, ni d'ailleurs dans les ateliers - de moindre importance - du Vercors). Malgré tout, ne pourrait-on envisager que cette teinte, finalement pas très différente de celle d'un alliage cuivreux - bien astiqué ou un peu patiné selon les cas - ait joué un rôle, certes subsidiaire par rapport aux potentialités mécaniques du matériau mais peut-être non négligeable, dans la manière dont ces productions, indiscutablement de prestige, furent appréciées à une époque où le métal - le vrai - faisait figure de matériau d'avant-garde quasi-inaccessible? (pour la petite histoire, rappelons-nous le premier couvert en aluminium qui fut solennellement offert à Napoléon III ou les folies auxquelles les élégantes consentaient il y a un demi-siècle pour obtenir une paire de bas en nylon...).

Tout phantasme mis à part, cette belle plaquette fournit, sous une forme commode et attrayante ce qui ne gâte rien, une précieuse documentation sur des productions et des diffusions jusqu'ici bien difficiles à documenter depuis la France.

Chancerel, A., Marcigny, C. et Ghesquière, E. (dir.). 2005 - Le plateau de Mondeville (Calvados) : du Néolithique à l'àge du Bronze, Paris, éditions de la Maison des Sciences de l'Homme, coll. "dAf ", 99, 208 p.

Le plateau de Mondeville, situé dans la plaine de Caen, a fait l'objet de multiples opérations d'aménagement, précédées de diagnostics et de fouilles préventives entre 1990 et 1995. Trois zones industrielles y ont été implantées sur une emprise géographique relativement limitée : la ZAC du Marché d'Intérêt Régional (MIR), la ZAC de l'Étoile et la ZI de Mondeville-sud. Une dizaine de responsables d'opération se sont succédés sur les différents chantiers archéologiques au cours du temps. Par la suite, la coordination scientifique mise en place en vue d'une publication des sites fouillés n'a été rassemblée que très tardivement. Ces deux facteurs augurent à eux seuls des énormes difficultés rencontrées pour le travail de récolement des données. Autant dire qu'Antoine Chancerel, Cyril Marcigny et Emmanuel Ghesquière ont réalisé un véritable tour de force en rassemblant et harmonisant une documentation éparse et inégale, parfois fort lacunaire, en vue de la publication des résultats issus des différentes opérations préventives effectuées. Bien que publié en 2006, l'ouvrage a été rédigé entre 1994 et 1999 (Cf. Avertissement, p. 12). Une bibliographie actualisée permet d'estomper les décalages dus à l'avancée de la recherche entre la date de fin de rédaction et celle de la publication. On ne peut que féliciter les auteurs pour l'étendue du travail accompli et souligner la qualité formelle toujours aussi remarquable des publications de la collection dAf.

Louvrage s'ouvre sur un chapitre introductif (ch. 1 : Présentation générale des sites), à la suite duquel les différents sites archéologiques sont présentés les uns après les autres dans des chapitres successifs respectant la chronologie, de la phase néolithique VSG à la transition Bronze/Fer. Les chapitres sont de longueur et d'importance variables, en fonction du potentiel archéologique offert par chaque structure ou ensemble fouillé. Chaque opération donne lieu à une présentation détaillée des données collectées sur le terrain. Les mobiliers sont étudiés de façon méthodique et sont très bien documentés. Le texte, toujours fluide, est soutenu en permanence par des illustrations variées et de grande qualité, ainsi que par de nombreux tableaux et graphiques. L'étude des mobiliers lithiques, par exemple, est systématiquement synthétisée sous forme de tableaux à l'intérieur desquels apparait la totalité des pièces mises au jour. En bref, les données rassemblées lors des différentes opérations de fouilles sont offertes très généreusement aux lecteurs, donnant une impression de grande transparence 
dans la transmission des informations, ce qui n'a probablement pas toujours été simple.

Le Néolithique est évoqué à travers trois occupations différentes : une maison danubienne située au Haut-SaintMartin, dans la ZI sud (ch. 2), une série de fosses silos et une fosse d'extraction datées du Néolithique moyen II, égalerment dans la ZI sud (ch. 3) et une fosse à dépôts d'animaux datées du Néolithique récent dans la ZAC de l'Étoile (ch. 4). Pour cette période, les découvertes du plateau de Mondeville offrent un apport très positif et permettent de compléter les données régionales sur le courant VSG récent et sur le Néolithique moyen II.

La maison danubienne du Haut-Saint-Martin apparaît isolée, contrairement aux villages danubiens "classiques " qui présentent généralement des rangées de bâtiments très rapprochés. Les recherches de maisons mitoyennes n'ont donné aucun résultat. La maison et les fosses d'accompagnement sont bien datées, en particulier par l'outillage lithique en silex, qui place le site à la fin de la période VSG. Ce site complète les données sur la phase du VSG récent et confirme à nouveau la présence du modèle danubien dans l'ouest de la France. Les auteurs supposent d'ailleurs que l'extension des sites VSG pourra bientôt être vérifiée sur l'ensemble du territoire breton (p. 182).

L'occupation néolithique de la ZI sud se résume, quant à elle, à six fosses-silos et une fosse d'extraction, qui ont livré un corpus mobilier relativement important daté du Néolithique moyen II. Une datation ${ }^{14} \mathrm{C}$, supposée assez fiable, confirme une position immédiatement postérieure aux dates retenues pour le Cerny (p. 88). Ce chapitre donne lieu à une réflexion historiographique sur la "redécouverte » de l'ensilage et sur la fonction des silos (p. 56-58); on se prend à lire ce passage avec beaucoup d'intérêt alors que ces structures, pourtant si fréquentes dans les habitats, sont généralement évoquées de manière (assez) expéditive. Quoi qu’il en soit, le mobilier céramique et lithique rassemblé ici augmente considérablement les données régionales, particulièrement dans le domaine des ensembles céramiques domestiques. Ceci permet de préciser les affinités culturelles, fluctuantes au cours du temps et en fonction des mobiliers considérés, et qui ne correspondent pas forcément avec celles qui ont été identifiées dans le domaine de l'architecture funéraire (p. 88-92).

Le dernier site néolithique présenté, une fosse circulaire à dépôts osseux (un faon et un jeune bovidé, accompagné d'un fémur et d'un tibia de foetus humain), bien qu'assez singulier dans sa composition, n'offre que peu de prise pour une interprétation définitive. Sa datation s'appuie uniquement sur un ${ }^{14} \mathrm{C}$ effectué sur les ossements du faon (p. 95) : 3303-2898 $\mathrm{BC}$ cal., soit le Néolithique récent. Des découvertes nouvelles permettront peut-être un jour de mieux comprendre la vocation de ces fosses, a priori non funéraires comme le soulignent les auteurs, ayant reçu des dépôts animaux mêlés à quelques restes humains épars.

Les connaissances sur l'extrême fin du Néolithique et sur le début de l'âge du Bronze auraient peut-être pu avancer de manière significative grâce à la fouille de l'enclos de la ZAC du MIR (ch :5). Malheureusement, "Cette opération est incontestablement celle qui cristallise aujourd'hui le plus grand nombre de regrets" (p. 100). Et pour cause... L'enclos, grossièrement quadrangulaire et délimitant une surface de $1575 \mathrm{~m}^{2}$, a été exploré en un temps record (15 jours) et par une équipe plus que réduite (4 personnes). Ni les abords de l'enclos, ni l'aire centrale n'ont pu être décapés et cette dernière n'a pu être appréhendée qu'à l'aide de quelques tranchées transversales et fenêtres. En l'absence de données sur les surfaces mitoyennes, la fonction de cet enclos peut difficilement être précisée. Les auteurs tentent d'exploiter au maximum les données apportées par le mobilier qui témoigne de deux occupations successives dans le temps, l'une au Néolithique récent et l'autre au Campaniforme. Ils proposent d'interpréter la structure comme une enceinte néolithique, probablement associée à un habitat à l'origine et ayant fait l'objet d'une réoccupation et de réaménagements à l'époque campaniforme (p. 109-110).

S'il ne fallait retenir qu'un seul site découvert sur le plateau de Mondeville, il serait bien difficile de trancher entre l'enceinte de la ZAC de l'Étoile (ch. 6) et celle, double, de la ZI nord (ch. 7). La lecture de ces deux chapitres est tout à fait passionnante et apporte une nouvelle bouffée d'air frais dans un paysage régional de l'âge du Bronze moyen demeuré longtemps si métallique. Les deux sites donnent lieu à des études rigoureuses et d'une grande pertinence. La présentation de l'évolution pédogénétique des sols du site de Mondeville (p. 114-116), les réflexions sur les creusements et les processus de comblement des fossés (p. 119-120 et 143-145), de même que l'estimation du cubage et donc du temps de travail nécessaire pour la réalisation d'un fossé équivalant à celui de l'enceinte sud de la ZI nord (p. 144) s'avèrent tout particulièrement stimulantes. L'enceinte de l'Étoile et les deux enceintes accolées de la ZI nord présentent des faciès étonnamment comparables. Ces deux sites n'ont livré aucune trace d'occupation autre que les fossés, malgré un décapage relativement important de l'aire centrale et d'une petite partie des abords. Dans les deux cas, le mobilier céramique, uniformément attribuable au contexte Deverel-Rimbury, situe l'occupation à la fin du Bronze moyen et au début du Bronze final. Le remplissage de l'enceinte double de la ZI sud a livré trois objets en bronze (une épingle à renflement fusiforme nervuré, une hache à talon de type normand et une pointe de lance de type Sucy) qui précisent le contexte chronologique à la fin du Bronze moyen (XIV ${ }^{e}$-XIII ${ }^{e}$ siècles avant J.-C.). 
Les similitudes entre les deux sites se prolongent dans les résultats de l'étude du mobilier osseux. Celui-ci, dominé quantitativement par le bœuf, révèle de véritables singularités : importance relative du cheval parmi les espèces domestiques, faible part de la faune sauvage, présence de nombreux éléments de crâne et d'os longs non segmentés, présence d'ensembles anatomiques complets et de quelques restes humains épars. Ces caractéristiques s'éloignent d'un traitement boucher habituel et évoquent immédiatement des pratiques de dépôts qui sont rares sur les sites à vocation uniquement domestique. La présence d'une hache et d'une pointe de lance intactes dans des fossés d'enceinte contraste également avec d'autres rejets véritablement détritiques comme les vaisselles brisées. Ceci étant dit, l'interprétation la plus vraisemblable reste celle d'habitats ceinturés par une enceinte, à l'instar des quelques exemples comparables connus dans le sud de l'Angleterre et dont la fonction ne fait pas de doute.

Les deux chapitres suivants (ch. 8 et 9), consacrés à des structures isolées de la fin du Bronze final et du premier âge du Fer, laissent très rapidement place à une conclusion (ch. 10) qui remplit pleinement son rôle en replaçant les données récoltées sur le plateau de Mondeville dans un contexte régional plus large. À la lecture de ce livre, il paraît évident que les enceintes de l'Étoile et de la ZI nord vont rapidement devenir des références régionales pour le Bronze moyen, comme l'est déjà l'île de Tatihou publiée en 2003 par C. Marcigny et E. Ghesquière dans la même collection (dAf, 96). L'ouvrage cconsacré au plateau de Mondeville est une nouvelle pierre à l'édifice ambitieux du renouvellement de la recherche dans le domaine de l'occupation du territoire pré- et protohistorique de Basse-Normandie. Le dynamisme extrême des auteurs, dont on peine à suivre le rythme effréné des publications, contribue pleinement à la découverte, depuis quelques années déjà, d'un âge du Bronze normand dans lequel les dépôts métalliques ne sont plus les seuls et uniques témoins de la présence humaine. Espérons et gageons que de prochains grands décapages révèleront enfin prochainement des sites de même nature et de même ampleur dans le reste du Grand Ouest.

Jean-Laurent Monnier (CNRS, UMR 6566, université de Rennes 1)

MarCigny, C., GHesquière, E. ET Juhel, L., 2006 - Baligan. Les collections préhistoriques et gallo-romaines du Muséum Emmanuel-Liais, édition Ville de Cherbourg-Octeville, coll. " Unica », 76 p. (ISBN 2-900-481-25-2).

À l'heure où les muséums d'histoire naturelle « reviennent dans l'air du temps » (E. de Roux, Le Monde, 26 septembre 2007), c'est un vif plaisir pour un ancien naturaliste que de saluer, à travers la publication de sa série de guides « Unica » le dynamisme de l'établissement cherbourgeois (quinze titres sont annoncés, celui-ci étant le deuxième à sortir de presse). Le présent volume est, nous précise l'introduction, dédié à Baligan, le dragon qui hantait dit-on la grotte de Diélette et a copieusement nourri le riche légendaire du nord-Cotentin. Page suivante, les auteurs nous rappellent que la ville de Cherbourg possède deux splendides fonds patrimoniaux miraculeusement épargnés par la seconde guerre mondiale : le muséum Emmanuel Liais et la bibliothèque Jacques-Prévert.

Après cette "mise en bouche ", le cœur de l'ouvrage suit un plan classiquement chronologique, en ne craignant pas de rappeler en tant que de besoin quelques notions qui pourront apparaittre comme des truismes pour le spécialiste - mais n'oublions pas que nous sommes ici dans un ouvrage de grande diffusion. À chaque subdivision chronologique, les auteurs prennent donc la peine (et parfois le risque) de donner des dates chiffrées, bien utiles pour permettre au profane de se situer (dommage par contre que la " chronologie générale " (p. 63) n'ait pas été plus travaillée). Bien dans cette logique de vulgarisation de bon aloi, le texte est clair et fluide mais desservi par une présentation un peu terne (typographie et mise en page). Cependant, la parcimonie des illustrations intexto est compensée par trois magnifiques planches hors-texte en quadrichromie, dont un dépliant central qui nous propose une somptueuse restitution du site mésolithique d'Auderville où le graphisme épuré de L. Juhel fait merveille pour retravailler un panorama photographique.

La matière régionale étant ce qu'elle est, la partie chronologique s'ouvre sur le Mésolithique qui, pour une fois, a la part belle dans un ouvrage de ce genre : 11 pages en détaillent les trois grandes phases attestées régionalement, avec présentation des sites de référence. Le Néolithique est ensuite exposé sous ses différents aspects avec évocation de sa composante mégalithique, injustement méconnue dans le nord-Cotentin malgré les inventaires réalisés ces dernières années par deux des auteurs (E. G. et C. M.) accompagnés par H. Lepaumier (et injustement passés sous silence dans la bibliographie car restés à l'état de " littérature grise »). Ce chapitre se clôt par une brève évocation du Campaniforme (ici traité à la suite du Néolithique, sans doute en raison du manque d'associations métalliques connues régionalement).

La présentation de l'âge du Bronze fournit l'occasion de rappeler les affinités armoricaines de la région avec l'évocation du tumulus de la Fosse-Yvon réétudié il y a une 